\section{Viscosity of Liquid Helium II}

IN recent experiments on the flow of liquid helium II through very small openings, carried out by Allen and Misener', and by Kapitza ${ }^{2}$, an upper limit to the viscosity was placed at $10^{-9}$ c.G.s. units, in definite disagreement with previous results obtained in this laboratory by Wilhelm, Misener and Clark ${ }^{3}$ by means of an oscillating cylinder. However, in none of these experiments were conditions such as to ensure laminar flow.

In view of this apparent extremely rapid flow of helium II, we thought that a study of the flow as the liquid passed through the $\lambda$-point would give some information concerning the rate of transformation from helium II to helium I. For this purpose, a capillary tube $0.02 \mathrm{~cm}$. in diameter was filled to a length of $3 \mathrm{~cm}$. with washed emery powder, and connected to a reservoir. In order to prevent the fountain effect observed by Allen and Jones ${ }^{4}$ with a similar device, the emery plug was protected from

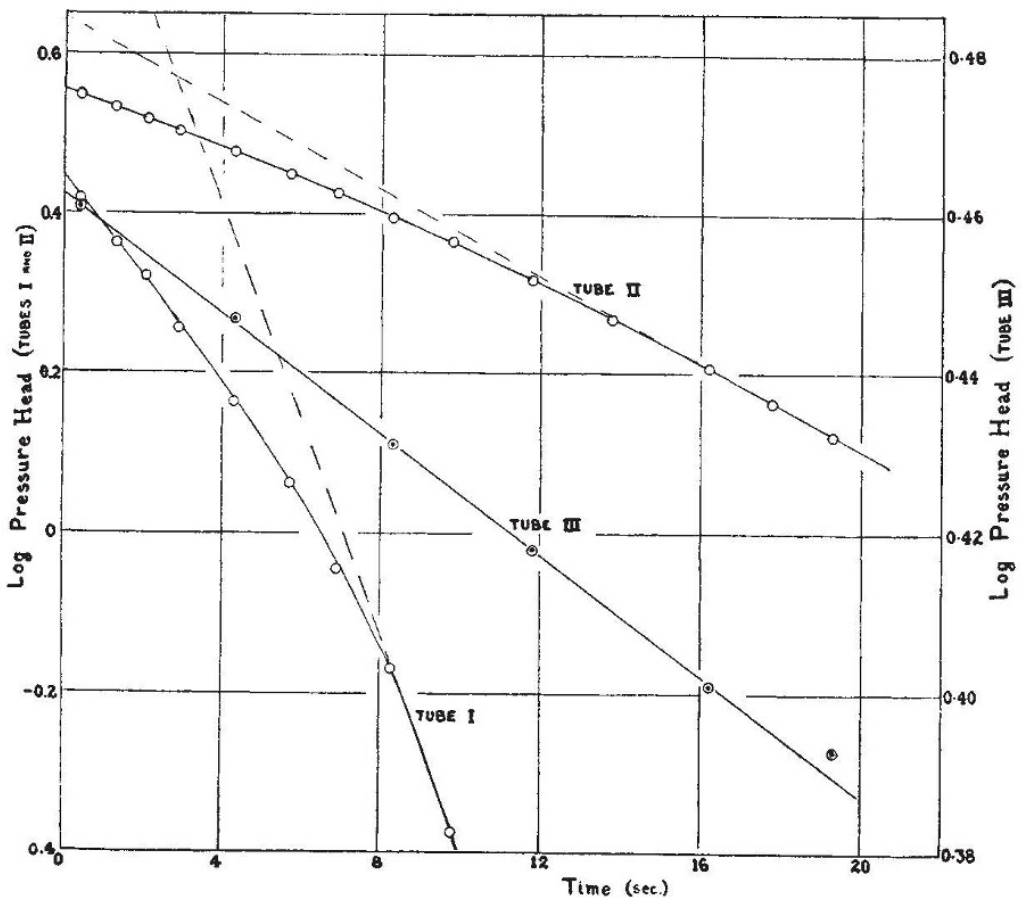

In no case did the results suggest that the liquid helium II was behaving as a 'super-fluid'. In the accompanying diagram, the logarithm of the pressure head is plotted against the time, for a series of measurements at $2 \cdot 16^{\circ} \mathrm{K}$. It will be seen that, for the slower rates of flow, the relation is linear, showing that the rate was nearly proportional to the pressure. The viscosity has been calculated from these results, and is given in the accompanying table. The values

\begin{tabular}{|c|c|c|c|c|c|}
\hline Tube & $\begin{array}{l}\text { Diameter } \\
\text { (cm.) }\end{array}$ & $\begin{array}{l}\text { Length } \\
\text { (em.) }\end{array}$ & $\begin{array}{c}\text { Viscosity } \\
\text { (c.G.s. units) }\end{array}$ & $\begin{array}{l}\text { Velocity } \\
\text { for small } \\
\text { pressure } \\
\text { heads } \\
\text { (cm./sec.) }\end{array}$ & $\begin{array}{l}\text { Reynolds } \\
\text { number }\end{array}$ \\
\hline $\begin{array}{r}\text { I } \\
\text { III }\end{array}$ & $\begin{array}{l}0 \cdot 035 \\
0.024 \\
0.013\end{array}$ & $\begin{array}{l}1 \cdot 8 \\
1 \cdot 9 \\
1 \cdot 7\end{array}$ & $\begin{array}{l}7.9 \times 10^{-5} \\
7.8 \times 10^{-5} \\
5.4 \times 10^{-5}\end{array}$ & $\begin{array}{l}21 \\
28 \\
13\end{array}$ & $\begin{array}{l}750 \\
670 \\
170\end{array}$ \\
\hline
\end{tabular}

quoted are correct in order of magnitude only, since the tubes were not very uniform, and no end corrections were made. Using this estimate of the viscosity, the Reynolds number has been calculated, and in all three cases is less than 1,000 for the smallest velocity measured. We hope, by using more uniform tubes, to obtain more accurate values for the viscosity.

These results are not compatible with those of Allen and Misener, or of Kapitza, but agree in order of magnitude with the earlier work of Wilhelm, Misener and Clark. We suggest that in the case of the long, fine capillary used by Allen and Misener, the observations can be explained by the transport phenomenon studied by Daunt and Mendelssohnn. Accord. ing to the results reported by the latter, the level in the reservoir used by Allen and Misener would have fallen at a rate of about 0.02 $\mathrm{mm}$. per second at $2 \cdot 17^{\circ} \mathrm{K}$. due to transport over the surface of the tube. This would account for the observed drop, and it seems probable that the actual flow through the long capillary was negligible. This would also account for the fact that the velocity did not vary greatly

radiation. In spite of the limit of viscosity reported by Allen and Misener, and by Kapitza, the flow of helium II through this tube could scarcely be detected. After trying several smaller plugs, short capillaries alone were used, of three different dia. meters. These were attached to reservoirs $0 \cdot 4 \mathrm{~cm}$. in diameter, which were fastened side by side to a millimetre scale, and arranged so that they could be raised or lowered in the liquid helium bath. To measure the rate of fall of the surfaces, a motion picture camera was used, and arranged to photograph the tubes and a stop watch simultaneously. In the first experiments the tubes were lowered into helium II at a temperature a little below the $\lambda$-point, and the flow into the tubes was observed as the temperature was slowly raised through the transformation to helium I. However, the change in the rate of flow on passing through the $\lambda$-point was not greatly marked, and so measurements at fixed temperatures in helium II were made. with the pressure head, and for the large increase in velocity at the lower temperature. In the experiments described here, the change in level due to this transport would have amounted to only about $0.1 \mathrm{~mm}$. during the time of the experiment, less than 1 per cent of the observed change. On the other hand, the rates of flow observed by Allen and Misener with a larger tube and reservoir, and that observed by Kapitza, seem too large to be fully accounted for by the surface transport, and further experiments on both the flow and the transport are highly desirable.

The work described above was carried out by $\mathbf{H}$. E. Johns, J. O. Wilhelm, and Dr. H. Grayson Smith.
McLennan Laboratory,
University of Toronto.
E. F. Burton.
(Director.)

${ }^{2}$ Allen, J. F., and Misener, A. D., NATURE, 141, 75 (1938).

2 Kapitza, P., NATURe, 141, 74 (1938).

'Burton, E. F., NATURE, 185, 265 (1935); Wilhelm, J. O., Misener, A. D., and Clark, A. R., Proc. Roy. 'Soc., A, 151, 342 (1935).

- Allen, J, F., and Jones, H., NATURE, 141, 243 (1938).

Daunt, J. G., and Mendelssohn, K., NATURE, 141, 911 (1938). 